\title{
Clustering Kinetics of Granular Media in Three Dimensions
}

\author{
Shiyi Chen ${ }^{1,2}$, Yuefan Deng ${ }^{3}$, Xiaobo $\mathrm{Nie}^{1}$ and Yuhai $\mathrm{Tu}^{2}$ \\ ${ }^{1}$ Center for Nonlinear Studies, Los Alamos National Laboratory, Los Alamos, NM 87545 \\ ${ }^{2}$ IBM Research Division, T. J. Watson Research Center, P.O. Box 218, Yorktown Heights, NY 10598 \\ ${ }^{3}$ Department of Applied mathematics and Statistics, SUNY at Stony Brook, Stony Brook, NY 11794-3600
}

\begin{abstract}
Three-dimensional molecular dynamics simulations of dissipative particles $\left(\sim 10^{6}\right)$ are carried out for studying the clustering kinetics of granular media during cooling. The inter-connected high particle density regions are identified, showing tube-like structures. The energy decay rates as functions of the particle density and the restitution coefficient are obtained. It is found that the probability density function of the particle density obeys an exponential distribution at late stages. Both the fluctuation of density and the mean cluster size of the particle density have power law relations against time during the inelastic coalescing process.
\end{abstract}

83.10.Pp, 83.70.Fn, 81.05.Rm

To understand the fundamental differences of macroscopic behaviors between a regular gas and the inherently dissipative granular media is a challenging problem. Recent studies [1] have revealed that inelastic collision in granular media gives rise to a variety of complex phenomena which are absent in classical ideal gas, including the formation of clustering structures [2 5] and collapsing [6,7]. To understand the clustering kinetics and to make the connection between the microscopic and macroscopic properties of granular media are not only crucial for constructing the constitutive equations of granular materials, but also important for various engineering applications, including packing [8], size segregation [9, 10] and transport of granular materials [1, 11].

Cluster formation has been studied before. In particular, using a model of hard disks with dissipation, Goldhirsch and Zanetti [2] found that the clustering particles form long string-like structure in two dimensions. Experimentally, two dimensional cluster formation has been studied in a driven system by Kudrolli et al. [4] using a system consisting of small steel spheres rolling on a smooth bounded rectangular surface with moving side walls. They measured the probability density functions (PDF) of the particle density and the particle velocity for the clusters near the driven wall. However, most existing numerical simulations and experiments for clustering dynamics are carried out for two dimensions and only a small number of granular particles (in the order of 10,000) are used. For such a system, it is difficult to obtain reliable statistics and to address problems connecting macroscopic description with microscopic dynamics.

In this Letter, we present an analysis of threedimensional clustering kinetics of granular media in a free cooling condition using a recently developed three-dimensional molecular dynamics (MD) code based on Massage-Passing-Interface (MPI). This parallel code makes it possible to simulate over one million dissipative particles in three dimensions and therefore allows us to tackle problems which are difficult in previous studies, in- cluding the growth of fluctuation density and the mean cluster size of the particle clusters. In this paper, we focus on studying the energy decaying dynamics, threedimensional structures of clusters and the scaling kinetics of the particle density.

The granular media in this paper is treated as a collection of identical spherical particles with radius $R$. The motion of each particle is simulated by Newton's second law while ignoring the angular motion. To specify the interaction forces in our MD simulation model, we assume that there are no interactions when particles are not in contact. The following interaction forces are included when two particles, $i$ and $j$ overlap [12], i.e., the distance $\left|\mathbf{r}_{i j}\right|\left(\mathbf{r}_{i j} \equiv \mathbf{r}_{j}-\mathbf{r}_{i}\right)$ is smaller than 2R: (1) An elastic restoration force: $f_{e l}^{(i)}=Y m_{i}\left(\left|\mathbf{r}_{i j}\right|-2 R\right) \mathbf{r}_{i j} /\left|\mathbf{r}_{i j}\right|$; (2) dissipations due to the inelasticity of the collision: $f_{\text {diss }}^{(i)}=-\gamma m_{i} \mathbf{v}_{i j}^{n}$ and $f_{\text {shear }}^{(i)}=-\gamma_{s} m_{i} \mathbf{v}_{i j}^{t}$. Here $Y$ is the Young's modulus, $m_{i} \sim R^{3}$ is the mass of particle $i$; $\mathbf{v}_{i j}^{n}=\left(\mathbf{v}_{i j} \cdot \mathbf{r}_{i j}\right) \mathbf{r}_{i j} /\left|\mathbf{r}_{i j}\right|^{2}$ and $\mathbf{v}_{i j}^{t}=\mathbf{v}_{i j}-\mathbf{v}_{i j}^{n}$ are the projections of the relative velocities $\mathbf{v}_{i j}\left(\equiv \mathbf{v}_{i}-\mathbf{v}_{j}\right)$ on the $\mathbf{r}_{i j}$ direction and the tangential direction, respectively; $\gamma$ and $\gamma_{s}$ are the dissipation coefficients for the relative motion of particles in the normal and tangential directions.

Initially particles are distributed uniformly in a cubic box with a small random perturbation. The initial particle velocities are drawn from an isotropic Gaussian distribution with variance $\sigma$. For simplicity, in this paper we only report results for $\sigma=4$ and the Young's modulus $Y=10^{5}$. The radius of granular particles, $R$, and the mass of the particles, $m$, are normalized to be 1 . A second-order scheme is used for the time integration of the motion of particles and simulations are carried out in a periodic box of size $L^{3}$. Because of the large value of Young's modulus $Y$, our time step $\Delta t$ has to be set to $\Delta t=4.0 \times 10^{-4}$, small enough to resolve the full collision process. The particle volume fraction $\alpha$ is defined as $\alpha=4 \pi N / 3 L^{3}$, where $N$ is the particle number in the system. The restitution coefficient, $r$, defined as the ratio of the relative speeds after collision and before col- 
lision, can be determined as: $r=\sqrt{\left(r_{n}^{2}+2 r_{s}^{2}\right) / 3}$, where $r_{n}=\exp \left(-\pi / \sqrt{\omega^{2} / \eta^{2}-1}\right), r_{s}=r_{n}^{2 \gamma_{s} / \gamma}, \omega=\sqrt{Y / m}$ and $\eta=\gamma / 2 m$.13.

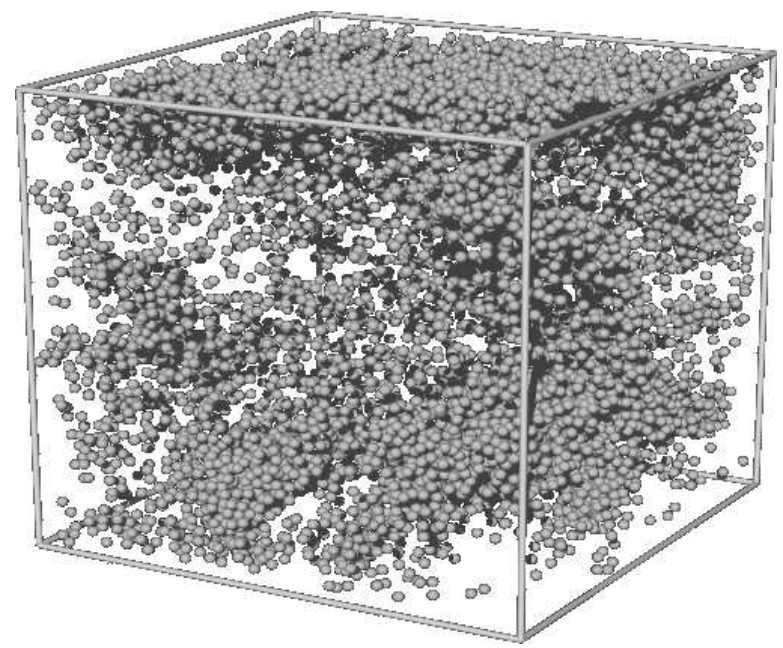

FIG. 1. A typical particle configuration at late stages of clustering formation for parameters $\alpha=0.074$ and $r=0.6$. The simulation is for $10^{6}$ particles, but only one-sixty-fourth of the simulation domain is shown.

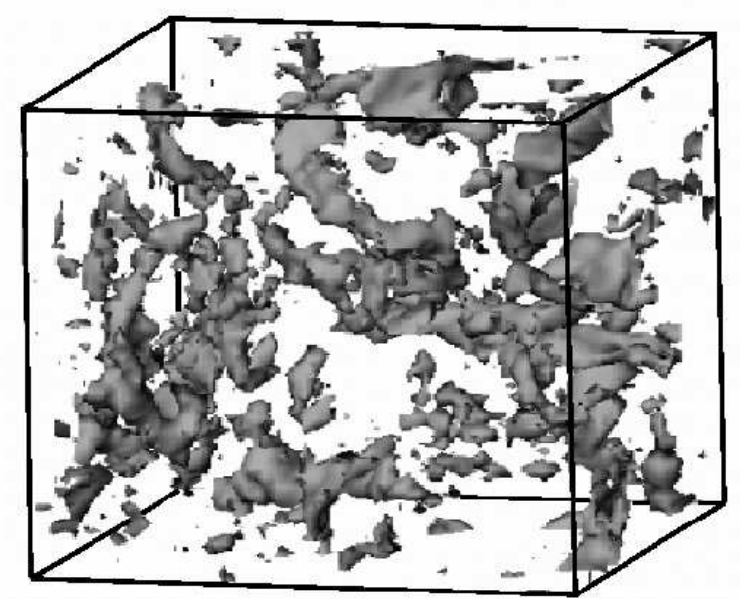

FIG. 2. Iso-surface of the particle density for $\rho=3.5 \rho_{m}$. The resolution in this plot is $50^{3}$ and the simulation was carried out for $L=384$.

In Fig. 1, we show a snapshot of a three dimensional particle distribution in a cooling granular system at late stage $t=1000$ from a simulation of one million particles (the initial characteristic collision time is of $O(1)$ ). The parameters in this calculation are $\alpha=0.074, \gamma=102$ and $\gamma_{s}=51$, which result in $r=0.6$. For clarity, only onesixty-fourth of the whole simulation domain is shown in this plot. To calculate the local particle density $\rho(\mathbf{x})$, we equally divide the whole space into small sub-boxes and average the particle number in each box. In Fig. 2, we present iso-surfaces of the particle density function $\rho(\mathbf{x})$ for $\rho=3.5 \rho_{m}$, where $\rho_{m}$ is the mean density of the whole space. From Fig. 1 and Fig. 2, it is evident that the particle clusters are formed and are more or less spatially inter-connected. In addition, the cluster structures are tube-like, which is to be compared with the string-like particle structure in two dimensions [2]. We have also calculated fractal dimensions for different regions using the box-counting method 14. We found that the fractal dimension $d_{f} \simeq 1.3$ for $\rho \geq 3.5 \rho_{m}$, in a qualitative agreement with the observed tube-like structure in Fig. 2, and $d_{f} \simeq 2.2$ for $\rho \geq \rho_{m}$.

In a cooling system, the forms of the particle motion fall roughly into two categories: free streaming and collision. During the streaming process, the speed of each particle is constant. The collision step decreases the speed of particles involved in the collision due to dissipation and reduces the energy. Based on a kinematic argument, it has been shown in [2,15] that if the spatial particle distribution is uniform for which the mean free path is proportional to $E^{1 / 2}$, and the local velocity distribution is random which leads to strong collision with loss of energy proportional to $E$, then the total energy of the system, $E(t)=1 / 2 \sum_{i} \mathbf{v}_{i}^{2}$, decays as:

$$
E(t)=\frac{E(0)}{\left(1+t / t_{c}\right)^{\beta}},
$$

where $t_{c}$ is a crossover time, and the exponent $\beta$ equals 2.

Our simulations show that in general the above equation can be used to describe the decay of the total energy during the particle clustering process, but $\beta$ depends on the properties of the granular media, in particular, the particle volume fraction and the restitution coefficient. Fig. 3a shows the energy decay exponent $\beta$ as a function of the particle volume rate $\alpha$ for the restitution coefficient $r=0.43$. In the inset, the total energy versus time for a typical case is shown. One can see that a power law decay can be identified for the late stage. Similar results are shown in Fig. $3 \mathrm{~b}$ for $\beta$ against the restitution coefficient $r$ with $\alpha=0.09$. The results shown here give a quantitative description of the dependence of the decay exponent on various material parameters: in the case of low density or large restitution, the particle distribution in the system is almost uniform and the resulting decay exponents equal 2, in a good agreement with the heuristic argument in [2,15. The smaller decay exponents are seen for systems with high densities and small restitutions, where clustering occurs. In this circumstance, the speed of particles in a cluster is approximately the same and the collision frequency is relatively low, leading to less energy dissipation and smaller energy decay rate.

The decrease of the energy decay rate has been studied previously in [2,5] using a linear hydrodynamic analysis based on phenomenological macroscopic equations. It is argued that for a system with particle clusters, the hydrodynamic modes have low decay rates. Quantitatively, it is interesting to notice that the energy decay exponent $\beta$ is round $1.4 \sim 1.5$ in the high density and small restitution regimes. To understand this nontrivial exponent, 
we decompose the total energy: $E(t)=E_{k}(t)+E_{f}(t)$, with $E_{k}(t)$ and $E_{f}(t)$ being the local spatially averaged kinetic energy and the fluctuation energy, respectively. We argue that in the high density or small restitution case, due to the formation of particle clusters, the motion of the clustered particles dominates, therefore the decay rate of $E(t)$ follows directly the macroscopic hydrodynamic decay of $E_{k}(t)$ :

$$
\begin{aligned}
E_{k}(t) & \sim \int|\vec{v}(\vec{k}, t=0)|^{2} \exp \left(-2 \nu|\vec{k}|^{2} t\right) d^{D} \vec{k} \\
& \sim t^{-D / 2},
\end{aligned}
$$

where $\nu$ is some effective viscosity and $D$ is the spatial dimension. In deriving (2), We have assumed that the initial velocity fluctuation $\vec{v}(\vec{k}, t=0)$ is independent of $\vec{k}$ for small $|\vec{k}|$ (certainly true for the initial velocity distribution). For $D=3$, Our numerical results of $\beta \sim 1.5$ in the clustering regime agree well with Eq. (2). To further verify our prediction, we have also carried out $2 \mathrm{D}$ simulations (not shown here). We found that the energy decay rate at high densities is indeed close to 1 , while the exponent $\beta$ for very low density remains 2 .
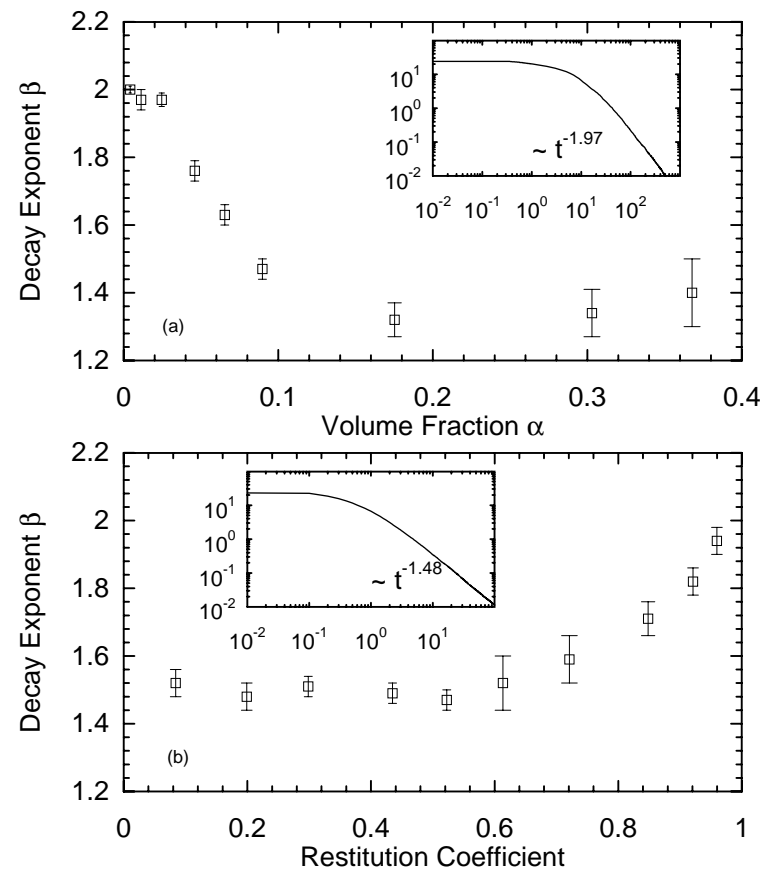

FIG. 3. The energy decay exponent $\beta$ as functions of the particle volume fraction (a) and the restitution coefficient (b). The inserted pictures show the typical energy decays against time for (a) $\alpha=0.011$; (b) $r=0.20$.

In the absence of clustering, the spatial particle distribution should be uniform and the PDF of the particle density is given by a $\delta$-function. On the other hand, if clustering occurs, particles coalesce to certain regions while leaving others void, leading to the disparity of particle density distribution. The study of the time dependence of the PDF of the particle density greatly enhances our knowledges of the clustering process and the characteristics of the clusters. It is interesting to note that the shape of the PDF is correlated with the energy decay exponent $\beta$. For $\beta \simeq 2$, the corresponding $\mathrm{PDF}$ of the density shows very little spreading, signaling the nonexistence of clustering in that parameter range, whereas in the clustering parameter regime (e.g., the parameters used in Fig. 4), $\beta$ is close to 1.5. In Fig. 4 , we present the PDF of the density function $\rho$ at different times with the same parameters as in Fig. 1. The statistics are obtained using $32^{3}$ sub-boxes. The density is normalized by the average density $\rho_{m}$. The PDF at $t=0$ is centered at one narrow density interval due to our specific initialization of the particle distribution. As the systems evolves, the PDF of the density function widens, implying the non-uniformity of the spatial particle distribution and the formation of clusters. Finally, the system becomes totally spread with more samples at lower density and fewer at high density. The PDFs at late stages can be approximated by an exponential function, indicating the existence of all possible densities. No local peak of PDF at high density was observed, at least for the simulation times we have reached, implying no specific characteristic density scale. Finally, in the inset of Fig. 4, we show the density fluctuation $\delta \rho(t)$ as a function of time, which follows a power law for almost three decades in time:

$$
\delta \rho(t)^{2} \equiv\left\langle\left(\rho-\rho_{m}\right)^{2}\right\rangle \sim t^{0.55} .
$$

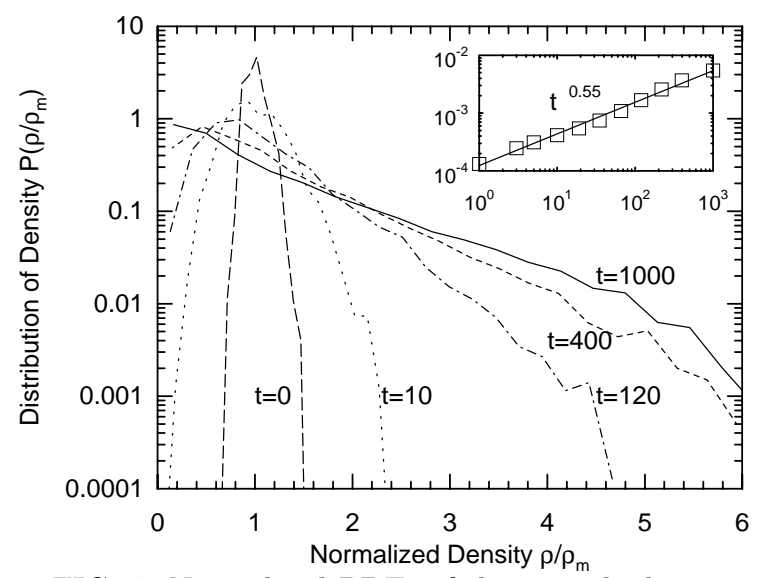

FIG. 4. Normalized PDFs of the particle density at different times. The inserted picture shows that the fluctuation of the density function grows as a power function of time.

To study the particle density correlation, we analyze the power spectra of the particle density function: $E_{\rho}(K)=\sum_{K-1 / 2<k<K+1 / 2} \rho(k) \rho^{*}(k)$, where $\rho(k)$ is the particle density in Fourier space. In the inset of Fig. $5, E_{\rho}(K)$ as a function of $K$ at different times are shown. Two features in the power spectra can readily be seen. First, as the time evolves, the peak value of the power spectra increases, in agreement with the inset in Fig. 4 due to the fact $\delta \rho^{2}=\int_{K} E_{\rho}(K) d K$. Second, if we denote $K_{\max }(t)$ for the wavenumber at 
which $E_{\rho}(K)$ has the maximum value, we can see that $K_{\max }(t)$ decreases as a function of time, indicating the increase of the typical cluster size. To quantify this behavior, we define a mean cluster size, $\lambda(t)$ by: $\lambda(t)=$ $\int_{K} E_{\rho}(K) d K / \int_{K} E_{\rho}(K) K d K$. In Fig. 5, we present $\lambda(t)$ as a function of time for the times when particles form clusters. Again, a power law dependence of $\lambda(t)$ on $t$ is observed over two decades in time [16]:

$$
\lambda(t) \sim t^{\zeta},
$$

where $\zeta$ is the growth exponent for the mean cluster size and is close to 0.13 for the given case. From other simulations not shown here, we note that $\zeta$ has a week dependent on the particle volume fraction and increases slowly with increasing of $\alpha$.

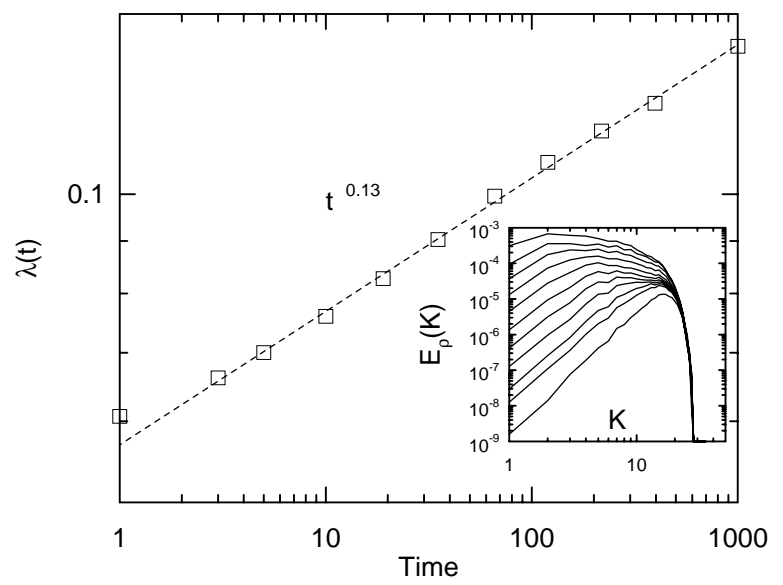

FIG. 5. The mean size of particle cluster as a function of time. In the inset shows the power spectra $E_{\rho}(K)$ at different times.

To summarize, we have studied the clustering phenomenon in the cooling process of three dimensional granular media. Large spatial density fluctuation is observed due to inelastic collision and the formation of the clusters strongly affects the energy decay rate. It is found that the total energy has a power law decay against time at late stages: $E(t) \sim t^{-\beta}$ with $\beta \sim 1.5$ in the parameter regimes where clustering forms. The value of $\beta$ crosses over to its mean field value $\beta_{M F T}=2.0$ for systems with large restitution constant $r$ or small density $\alpha$ where the particle clustering is absent. By making simple analogy to the decay of kinetic energy in fluids, we relate the value of $\beta$ in the clustering parameter regime to the spatial dimension $D: \beta=D / 2$. We observe that clusters in three dimensions form tube-like structures for high particle density regions at late stages. A preliminary study has revealed that the structure might be fractal. We have also studied the dynamical properties of clusters. It is found that the density fluctuation and the cluster size both obey power law scalings against time with nontrivial scaling exponents. We have no theory to explain the observed scaling dynamics and it is our hope that the present results would stimulate further studies in understanding these intriguing behaviors.

We thank Eli Ben-Naim and Gary D. Doolen for useful discussions. Numerical simulations were carried out at the Center for Scalable Computing Solution at IBM T. J. Watson Research Center using the SP machines.

[1] H. M. Jaeger, S. R. Nagel and R. P. Behringer, Physics Today, 32-38, April, 1996; H. M. Jaeger, S. R. Nagel and R. P. Behringer, Rev. Mod. Phys. 68, 1259 (1996).

[2] I. Goldhirsch and G. Zanetti, Phys. Rev. Lett. 70, 1619 (1993).

[3] J. J. Brey, M. J. Ruiz-Montero and D. Cubero, Phys. Rev. E. 54, 3664 (1996).

[4] A. Kudrolli, M. Wolpert and J. P. Gollub, Phys. Rev. Lett. 78, 1383 (1997).

[5] P. Deltour and J.-L. Barrat, Quantitative study of a free cooling granular medium, preprint, (1997).

[6] S. McNamara and W. R. Young, Phys. Rev. E, 50, R28 (1994).

[7] T. Zhou and L. P. Kadanoff, Phys. Rev. E, 54, 623 (1996).

[8] E. R. Nowak, J. B. Knight, E. Ben-Naim, H. M. Jaeger, and S. R. Nagel, Phys. Rev. E 57, 1971 (1998); E. BenNaim, J. B. Knight, E. R. Nowak, H. M. Jaeger and S. Nagel, Phys. Rev. E. preprint (1998).

[9] J. C. Williams, Power Technol. 15, 245 (1976).

[10] L. T. Y. M. Fan and F. S. Lai, Powder Technol. 61, 255 (1976).

[11] P. B. Umbanhowar, F. Melo and H. L. Swinney, Nature. 382, 793(1996).

[12] H. J. Herrmann, Computer simulation of granular media, in Disorder and Granular Media, ed. D. Bideau and A. Hansen, 1993 Elsevier Science Publishers.

[13] The connection between the restitution coefficient $r$ and the parameters of the granular media can be obtained through the following calculation: Let $x(t)$ and $y(t)$ be the relative coordinates along the $\mathbf{r}_{i j}$ direction and the tangential direction during two particle collision, we have: $m \ddot{x}+\gamma \dot{x}+Y x=0$ and $m \ddot{y}+\gamma_{s} y=0$. The solution of these equations gives the relative velocities after collision in these two directions: $-r_{n} \mathbf{v}_{i j} \cos (\theta)$ and $r_{s} \mathbf{v}_{i j} \sin (\theta)$. Then $r^{2}=r_{n}^{2} \cos ^{2}(\theta)+r_{s}^{2} \sin ^{2}(\theta)$. The average of $r^{2}$ over the relative angle $\theta \in(0, \pi / 2)$ leads to $r^{2}=\left(r_{n}^{2}+2 r_{s}^{2}\right) / 3$.

[14] Jens Feder, Fractal, 1988 Plenum Press, New York.

[15] S. McNamara, Phys. Fluids A. 5, 12 (1993).

[16] The deviation from the power law behavior at the early stage $t<5$ could be due to initial particle-particle interaction, which is different from the cluster-cluster interaction of the late stage growth. 